\title{
D-SEAMS: DEFERRED STRUCTURAL ELUCIDATION ANALYSIS FOR MOLECULAR SIMULATIONS
}

\section{A PREPRINT}

\author{
(1) Rohit Goswami* \\ Department of Chemical Engineering \\ Indian Institute of Technology Kanpur \\ rgoswami@iitk.ac.in
}

\author{
(1) Amrita Goswami ${ }^{\dagger}$ \\ Department of Chemical Engineering \\ Indian Institute of Technology Kanpur \\ amritag@iitk.ac.in
}

\author{
Jayant K. Singh ${ }^{\ddagger}$ \\ Department of Chemical Engineering \\ Indian Institute of Technology Kanpur \\ jayantks@iitk.ac.in
}

August 12, 2020

\begin{abstract}
Structural analyses are an integral part of computational research on nucleation and supercooled water, whose accuracy and efficiency can impact the validity and feasibility of such studies. The underlying molecular mechanisms of these often elusive and computationally expensive processes can be inferred from the evolution of ice-like structures, determined using appropriate structural analysis techniques. We present d-SEAMS, a free and open-source post-processing engine for the analysis of molecular dynamics trajectories, which is specifically able to qualitatively classify ice structures, in both strong confinement and bulk systems. For the first time, recent algorithms for confined ice structure determination have been implemented, along with topological network criteria for bulk ice structure determination. Recognizing the need for customization in structural analysis, d-SEAMS has a unique code architecture, built with nix, employing a YAML-Lua scripting pipeline. The software has been designed to be user-friendly and easy to extend. The engine outputs are compatible with popular graphics software suites, allowing for immediate visual insights into the systems studied. We demonstrate the features of d-SEAMS by using it to analyze nucleation in the bulk regime and for quasi-one and quasi-two-dimensional systems. Structural time evolution and quantitative metrics are determined for heterogenous ice nucleation on a silver-exposed $\beta$-AgI surface, homogenous ice nucleation, flat monolayer square ice formation and freezing of an ice nanotube.
\end{abstract}

Keywords structure-determination, analysis-engine, computational-chemistry, nix, lua, cpp

\section{Introduction}

The increase in power and efficiency of high-performance computing resources have enabled researchers to directly observe nucleation events in molecular-dynamics simulations. Concomitantly, the determination of ice-like structures from simulations is essential for the interpretation of nucleation events, since trajectories provide positional data of particles, and do not explicitly track crystal structures and defects [1]. Nucleation of soft-matter is further complicated by the emergence of competing ice polymorphs [2,3] with small free energy differences [4]. The ice-like structures formed are also continually distorted by thermal fluctuations, which locally disrupt long-range order. These issues can

\footnotetext{
${ }^{*}$ Currently at the Department of Chemistry, IIT Kanpur, equal contribution

${ }^{\dagger}$ equal contribution

${ }^{\ddagger}$ Corresponding Author
} 
make automated and accurate structural determination intractable, especially for weakly crystalline regions [5]. Surface interactions and confinement can strongly influence nucleation behaviour [6-8], which add to the complexity of the structure determination problem.

Water is a deceptively simple molecule, exhibiting rich and complex phase behaviour in bulk and confinement [9-19]. At least 17 bulk ice polymorphs have been observed experimentally [20,21]. Water confined within nanometer length scales exhibits even more diversity, forming ordered hydrogen-bond networks of ice nanotubes, monolayers, bilayers and trilayers [17, 22-28]. The structural determination of the several possible polymorphs of water, in bulk and in confinement, is of crucial importance in the qualitative and quantitative analysis of simulation data. The analysis of the evolution of ordered structures during a nucleation event is desirable, since it can reveal details of the underlying molecular mechanism and provide important physical insights into the system.

In this work, we present d-SEAMS, a cohesive post-processing structural analysis engine, which is capable of coherently classifying water structures under strong confinement and in bulk systems, from quasi-one and two-dimensional confinement to bulk ice polymorphs. Confined ice polymorphs are often identified "by eye" wherein the hydrogen-bond network (HBN) is manually inspected $[17,19,26]$. In particular, d-SEAMS automates the process of structural analysis and qualitative metric calculation, eliminating the need for visual inspection.

Conflicting package clashes due to transitive or indirect dependencies is a recurring problem in software development and use [29]. Resolving such issues can often be non-trivial, relying on removal of unnecessary dependencies, system updates (for most Linux clusters) and other manual strategies that may be ineffective for a complex dependency tree. These manual strategies are increasingly difficult to carry out on restricted access machines with high up-times, which are common to the HPC (High Performance Computing) clusters, used for such computationally demanding simulations.

d-SEAMS circumvents the problem of 'dependency hell' by using nix [30] for generating reproducible dependency build-graphs [31]. Users can run d-SEAMS using the exact build-environment of the developers and vice-versa, bypassing installation and use issues on various systems and HPC clusters, ensuring reproducible results.

The parameters of structural analysis techniques are often tweaked to suit the specific requirements of the desired study [32,33]. d-SEAMS has been designed to permit easy extensions to the code and the implementation of custom work-flows. The engine simultaneously incorporates user-friendly interface functions without compromising on functionality. Key features implemented include a primitive ring analysis algorithm [34], topological network criteria for bulk [35] and confined systems [36] and new qualitative order parameters. Popular analysis techniques, including bond-orientational parameters [32,37] and related criteria [38,39], have also been implemented. Outputs are produced in formats compatible with popular visualization softwares, including OVITO [40] and VMD [41]. We describe how d-SEAMS has been used to analyze and characterize ice nucleation on an ice-promoting AgI surface, homogenous bulk nucleation, the formation of monolayer ice and the freezing of a quasi-one-dimensional ice nanotube (INT).

\section{Code Architecture}

\subsection{Nix Expressions for Reproducible Builds}

Computational software implemented in declarative package management systems suffer from design concerns for the end-users and the developers. The developers have the onus to package their software as per the many imperative systems (Ubuntu and apt, RedHat and yum, ArchLinux and pacman). Additionally, the users must ensure that the interrelated dependencies match perfectly. In essence, the issue is that the packages and versions at the time of build are not guaranteed automatically at the user's end, even if the software is packaged appropriately for the operating system. This issue stems from the fact that the configuration after installation is the result of a series of stateful transformations which cannot be reproduced [42]. We have opted to package our software as a nix-derivation [30], which also provides a functionally reproducible environment for reproducible bug-tests. Since the build system is essentially a static graph of build actions, the environment produced is a boon for reproducibility, avoiding circular build-time dependencies and incomplete dependency specifications [43]. More details on the design rationale are in the Electronic Supplementary Information.

\subsection{Pipeline of Work-flows}

Figure 1 visually depicts the logical pipeline of typical work-flows in d-SEAMS. An input system can either be a bulk system, a quasi-one-dimensional system or a quasi-two-dimensional system. The criteria and analysis algorithms tend to differ across scales as well. Thus, we have separated the mutually exclusive work-flows for bulk, quasi-one-dimensional and quasi-two-dimensional systems, with separate modular code blocks for each type of system. The general software pipeline is organized as follows: 


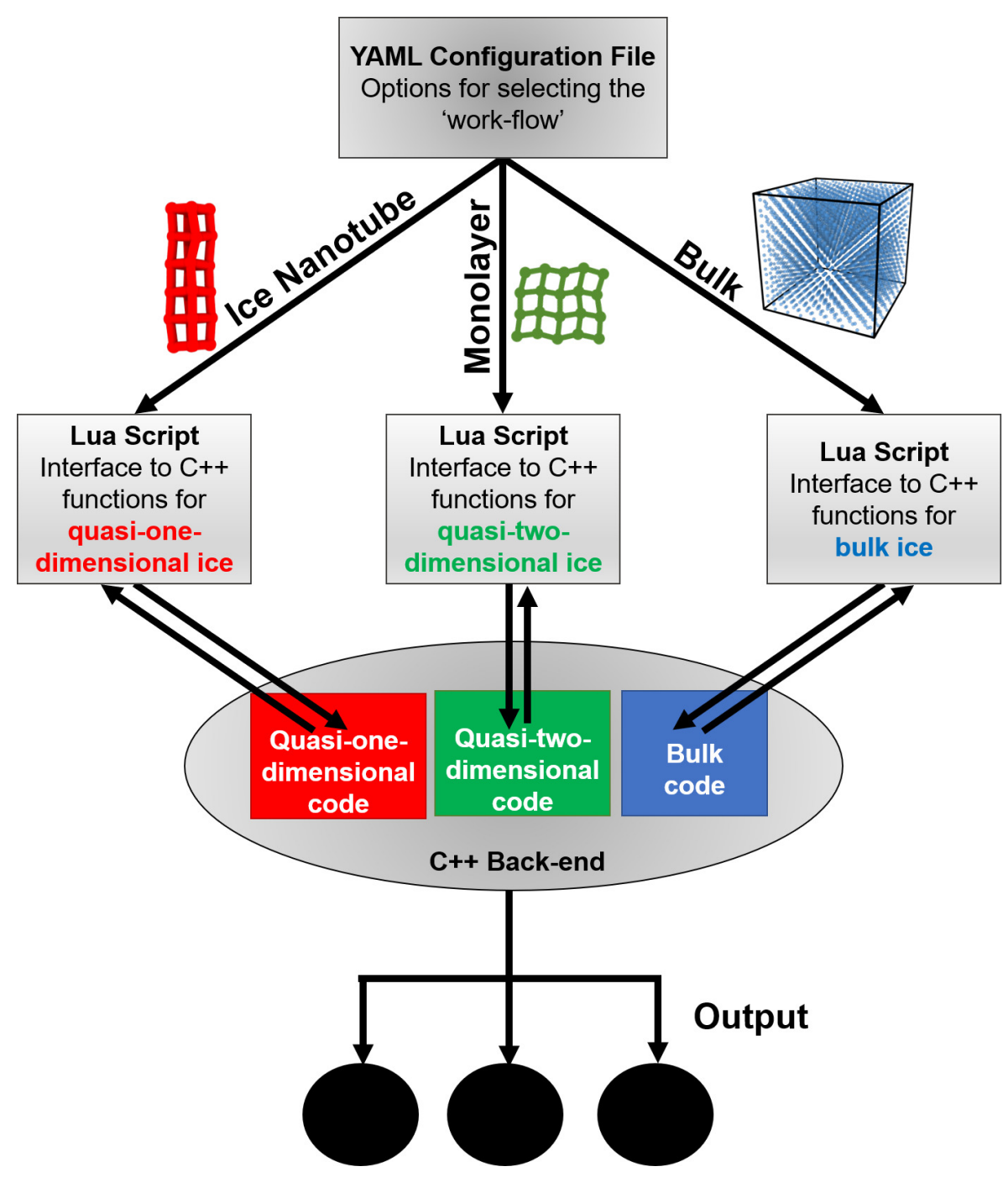

Figure 1: Code pipeline of d-SEAMS. The 'YAML' script file provides options to choose the type of the system and the corresponding work-flow, for either bulk, quasi-one-dimensional quasi-tqo-dimensional monolayer sytems. The 'Lua' interface functions interact with the ' $\mathrm{C}++$ ' functions for each work-flow, and functions of other work-flows are not exposed to the user.

- The user chooses whether the system is a bulk system, quasi-one-dimensional or quasi-two-dimensional system, by setting the appropriate values in the YAML input file.

- The Lua script calls the functions to read in, analyze and output the desired results.

- Simulation data is read from LAMMPS trajectory files. The XYZ file format is also currently supported. Other file formats may be converted into an ASCII LAMMPS trajectory format [44].

- Options for controlling the output results can be specified in the Lua file input.

- Output directories are created automatically, according to the chosen work-flow.

\subsection{Structural Identification Features}

We are able to apply arbitary transformations on the particle collections, in an efficient and idiomatic manner. Currently we have implemented the following structural schemes based on this easily extensible framework:

- The bond orientational parameters [32, 37], CHILL [38] and CHILL+ [39] parameters have been implemented. Clustering of ice-like molecules [45], based on these parameters can be optionally carried out by the user. The largest ice cluster so obtained can also be re-centered, for ease of visualization. 
- Topological network criteria for bulk ice determination [35] have been implemented. d-SEAMS is able to identify and write out detailed information about Double-diamond cages (DDCs), hexagonal cages (HCs), and mixed rings for every frame. Additionally, the number of basal and prismatic rings are also computed.

- Primitive rings are identified. First, all possible rings are found using an exhaustive backtracking algorithm, following which non-shortest path rings are removed [34]. Ring networks with only hydrogen-bonded connections can be optionally determined.

- Confined quasi-two-dimensional ice classification by topological and graph theoretic approaches to the hydrogen-bonded ice-like particles.

- Quasi-one dimensional ice nano-tube (INT) and quasi-two-dimensional monolayer ice classification via topological network criteria [36]. The building blocks of $n$-gonal prismatic ice are explicitly and unequivocally identified.

- d-SEAMS is capable of calculating geometric order parameters for describing the phase transitions in confined ice.

\section{Applications}

Using d-SEAMS, structural and qualitative information as a time series can be easily obtained. This makes it possible to study a variety of diverse nucleating systems. Even the mechanism of nucleation growth can be inferred from an ensemble of trajectories, by analyzing the qualitative metrics supported by d-SEAMS.

\subsection{Bulk Systems}

\subsubsection{Heterogenous Nucleation on an Ice-Promoting Surface}

Silver iodide is an effective ice nucleating agent, whose lattice closely resembles that of bulk ice [46]. The smooth Ag-exposed $\beta$-AgI surface has been known to promote layer-by-layer growth of hexagonal ice (Ih) and cubic ice (Ic) in simulations [47]. Using d-SEAMS, we probe the underlying mechanism of the ice nucleation growth and behaviour. Ten independent simulations of 5120 TIP4P/Ice [48] molecules on a free-standing AgI surface were run upto $\approx 200 \mathrm{~ns}$ at $240 \mathrm{~K}$. The simulation setup is similar to that of previous work in the literature [49].

Here we employ a topological network criterion [35] for identifying the building blocks of Ih and Ic, called hexagonal cages (HCs) and double-diamond cages (DDCs), respectively. Rings which belong to both HCs and DDCs are classified as mixed rings.

Figure 2 depicts layers of HCs and DDCs, growing from the AgI surface, after $200 \mathrm{~ns}$ of simulation time. The close lattice match between the AgI surface and the ice phases is clearly visible. However, the CHILL algorithm [38] identifies the first layer of molecules close to the surface as liquid. It was noted in previous studies that although the CHILL algorithm does not identify the first layer of water molecules as ice, these molecules actually form part of the initial ice layer $[47,50]$. The topological network criterion used here correctly classifies these water molecules as constituents of HCs or DDCs.

The Ag-exposed $\beta$-AgI surface supports the attachment of the basal plane of HCs, as well as DDCs. Figure 3 shows how layers grow through attachment of HCs and DDCs.

The peripheral rings of DDCs can support the attachment of both HCs and DDCs. However, the prismatic planes of an HC (highlighted in blue, green and mauve in Figure 3(b)) can only support the anchoring of HCs. Since the HCs grow upwards from the AgI surface, through the basal plane (highlighted in yellow in Figure 3(a)), only HCs can grow from the prismatic planes of pre-existing HCs in each layer. We surmise that the layer-wise growth of HCs in the lateral dimensions is because of this growth behaviour. This is in keeping with the stacking effects observed in the literature [49].

The effect of the surface is the most significant within the first two layers of cages. In all the independent trajectories, a single layer of either HCs or DDCs is formed first, upon which a second layer of DDCs grows. The two possible outcomes of the stacking of cages, observed in independent simulations, are depicted visually in Figure 4. Figure 4(a) shows the overhead view of the stacking arrangement when the first layer and second layer are HCs and DDCs, respectively. Figure 4(b) shows the other stacking arrangement observed, wherein the first and second layers are exclusively composed of DDCs. Both types of arrangements seem equally likely, based on the roughly equal proportions of stacking observed in all the independent simulations performed. Regardless of the composition of the first two layers, alternating layers of varying widths of HCs and DDCs are formed in all the simulations. Interestingly, although HCs sometimes grow on the first layer, by the end of the simulations, the second layer is exclusively composed of DDCs. 
(a)

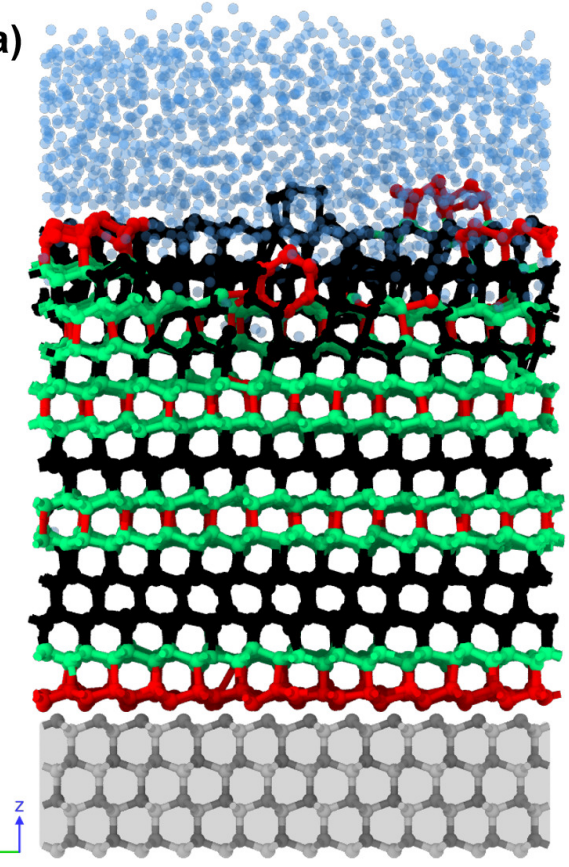

(b)

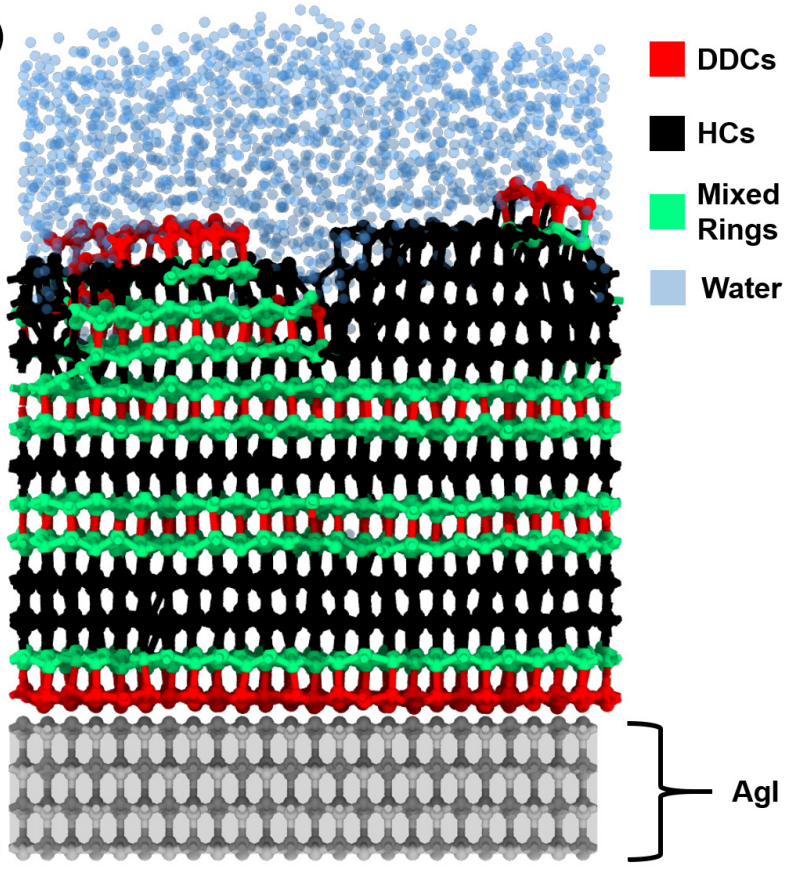

Figure 2: (a) $Y Z$-plane and (b) $X Z$-plane views of layered hexagonal ice (Ih) and cubic ice (Ic) after $200 \mathrm{~ns}$. DDCs, HCs and mixed rings are coloured in red, black and green, respectively. The silver and iodine atoms are dark-grey and grey respectively, with the AgI sheet highlighted in light grey.

We track the growth of the first two layers, with time, for a particular trajectory, whose stacking at the end of $200 \mathrm{~ns}$ is shown in Figure 4(a).

Figure 5 shows the growth of the first two layers of cages within the first $100 \mathrm{~ns}$ of simulation time. After $\approx 60 \mathrm{~ns}$, the second layer is partially composed of HCs and DDCs (Figure 5(b)). The HC portion is not anchored to the DDC part of the second layer, since DDCs cannot grow on the exposed lateral prismatic faces of the HCs. We note that eventually, by the time that the second layer of cages becomes continuous, HCs in the second layer disappear and are completely replaced by DDCs. In fact, the eventual layering of HCs and DDCs in the first two layers from the AgI surface is also observed on void-defect incorporated Ag-exposed $\beta$-AgI surfaces [49].

\subsubsection{Homogenous Nucleation: Growth of the Largest Ice Cluster}

Here, we have analyzed a trajectory which exhibits a successful homogenous nucleation event, in the bulk phase. Independent simulations of 4096 particles, modelled using the monoatomic water (mW) water model [51], were equilibriated at $300 \mathrm{~K}$ and quenched to $208 \mathrm{~K}$. Simulations were subsequently run at constant temperature and pressure, in the NPT ensemble, at $1 \mathrm{~atm}$ and $208 \mathrm{~K}$ for upto $1 \mu \mathrm{s}$. Figure 6 shows the structural features and growth of the largest ice cluster. In our ensemble of trajectories, we have observed that the largest ice crystallites which survive and grow to the post-critical size are rich in DDCs (shown in black in Figure 6). We find that even in post-critical crystallites, the core of the crystallites remains rich in DDCs. These findings match those gleaned from extensive forward-flux sampling simulations of both $\mathrm{mW}$ water and TIP4P/Ice [35]. The growth of the largest crystallite with time, showing fluctuations in the cluster size with time, is depicted in a supplementary movie, entitled largestClusterGrowth.avi, in the ESI (electronic supplementary information).

\subsection{Quasi-Two-Dimensional Systems}

We further analyze Flat Monolayer Square Ice (fMSI), using d-SEAMS. FMSI has been observed in simulations, when water subjected to a lateral pressure of $1 G P a$ is sandwiched between rigid graphene sheets, separated by a slit width of $6 \AA$ [26, 52]. d-SEAMS is capable of calculating the coverage area metric [36], an area-based order parameter, at every timestep of a trajectory. We demonstrate the efficacy of the coverage area metric in describing phase transitions, 
(a)

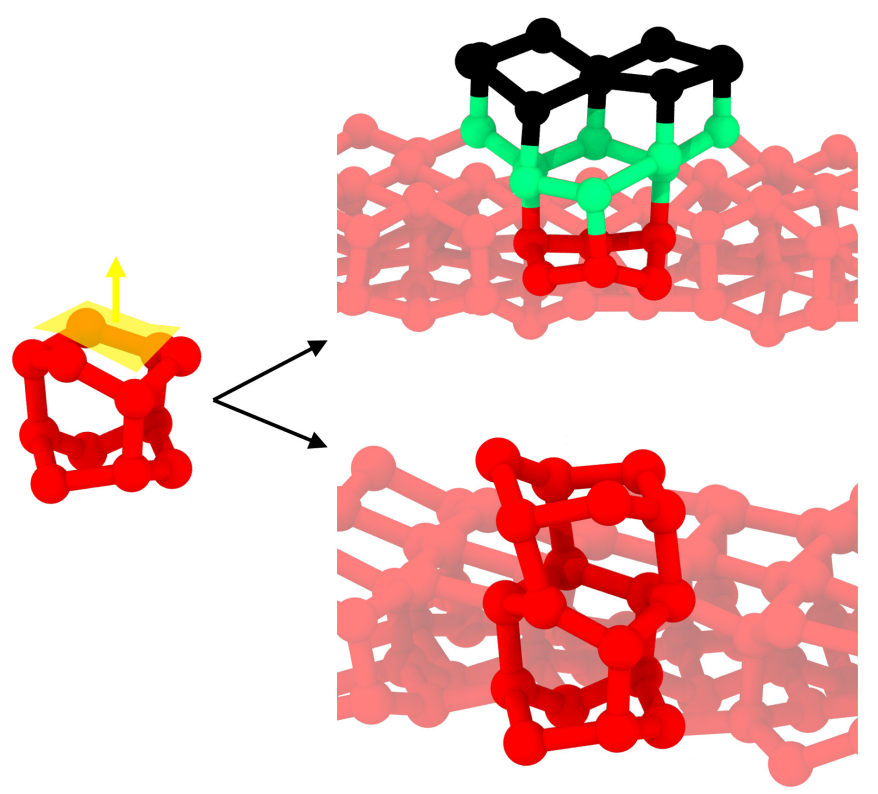

(b)

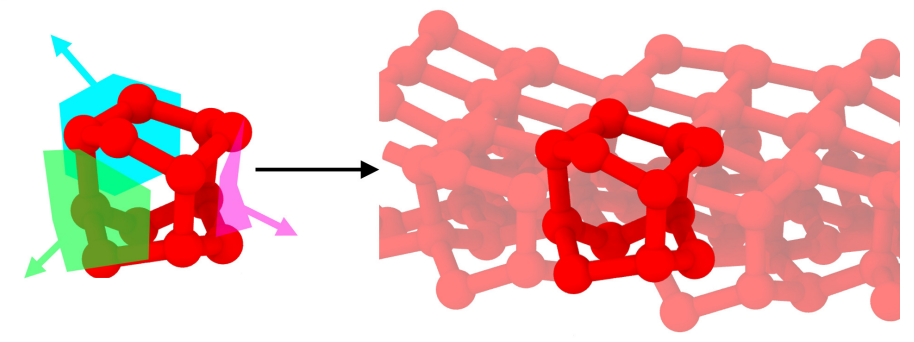

Figure 3: (a) Attachment of cages on the upper basal face of an HC. The basal face has been highlighted in yellow. Basal faces of an HC can support the growth of both DDCs (black) and HCs (red). (b) Attachment of HCs on the three prismatic faces, shaded in blue, green and mauve, of an HC. The prismatic faces of an HC cannot support the growth of DDCs. The colour scheme is the same as in Figure 2.

during the cooling of a quasi-two-dimensional monolayer from $320 \mathrm{~K}$ to $310 \mathrm{~K}$ over $1 \mathrm{~ns}$. The visuals produced were generated from the output of d-SEAMS. Figure 7 shows snapshots of the monolayer water through various stages of cooling, exhibiting a gradual increase in the proportion of the 4-membered rings. At $320 \mathrm{~K}$, the coverage area of the 4-membered rings is $\approx 70 \%$, which indicates that the monolayer is predominantly fMSI. This phase change is depicted in a supplementary video (monolayer . avi) in the ESI.

d-SEAMS is also capable of calculating the in-plane radial distribution function. The solid-like ordered nature of the ice formed at $310 \mathrm{~K}$ is typified by the shape of the 2-dimensional oxygen-oxygen radial distribution function in Figure 8. The $g_{O O}(r)$ has the highest peak at $2.775 \pm 0.05 \AA$, followed by a shoulder peak and a third smaller peak; the same features have been previously observed for fMSI. The frames were obtained from an equilibriated $1 \mathrm{~ns}$ run at a constant temperature of $310 \mathrm{~K}$ and lateral pressure of $1 \mathrm{GPa}$.

Table 1: Ring statistics and Coverage Area percentage for triangular and square ices

\begin{tabular}{ccc}
\hline Number of nodes & Coverage Area ${ }_{n} \%$ & Number of rings \\
\hline $\mathrm{n}=4$ & $74.422 \pm 3.345$ & $238.94 \pm 10.723$
\end{tabular}




\begin{tabular}{ccc}
\hline Number of nodes & Coverage Area ${ }_{n} \%$ & Number of rings \\
\hline $\mathrm{n}=3$ & $0.877 \pm 0.5$ & $6.2 \pm 3.544$ \\
\hline
\end{tabular}

Figure 1 summarizes the ring statistics and coverage area metric values, determined by d-SEAMS, for the fMSI structure formed at $310 \mathrm{~K}$. The coverage area metric is a more intuitive and more stable metric than the number of rings, as is evident from the data. The number of rings is more sensitive to small thermal fluctuations which are not relevant to the overall classification scheme.

\subsection{Quasi-One-Dimensional Systems}

The perspective views of ice nanotubes (INTs) are shown in Figure 9, for water constrained within a $(13,0)$ and $(11,0)$ zig-zag smooth featureless single-walled nanotube (SWNT). For the SWNTs, $R=13$ and $R=11$ correspond to diameters of $10.1 \AA$ and $8.6 \AA$, respectively.

At an axial pressure of $P_{z z}=0.1 M P a$, primarily hexagonal prism blocks are formed within the INT in Figure 9(a). A small proportion of the ice nanoribbon is comprised of pentagonal prism blocks, while the rest are deformed prism blocks classified as an 'unclassified' phase. The unclassified phase may denote deformed prisms, the liquid phase or hollow helices, which are not explicitly differentiated using the prism classification scheme. In this particular case, the hollow nature of the nanoribbon and the interspersed positions of the unclassified phase between prism blocks, indicate that the unclassified phase is comprised of deformed prisms.

Figure 9(b) shows the $(11,0) \mathrm{SWCT}$, subjected to an axial pressure of $P_{z z}=1 M P a$. Under these conditions, the INT is predominantly comprised of tetragonal prism blocks, with intermittent deformed prism blocks.

The length of the INT in Figure 9(a) is smaller than that in Figure 9(b). The number of prism blocks of each type is an unreliable indicator, which does not qualitatively describe the relative proportions of the prismatic ice phases. Although a volume-based metric has been proposed [36], an approximate metric based on the normalized number of prism blocks may be used as a reasonable approximation of relative proportion.

We have observed that the average height of each prism block remains relatively constant at a value of $\approx 2.845 \pm 0.07$ $\AA$, irrespective of the number of nodes in the basal ring $(n)$ and even the applied pressure $P_{z z}$. Therefore, it is possible to define a theoretical maximum possible number of $n$-gonal prism blocks, assuming that the entire SWCT height is filled with $n$-gonal prism blocks, each of height $\approx 2.845 \AA$. Since the average height of the prism blocks is independent of $n$, the theoretical maximum number of prism blocks is the same for all $n$. The theoretical maximum number of prism blocks $N_{\max }$ is thus:

$$
N_{\max }=\frac{H_{S W C T}}{h_{\text {avg }}}
$$

where $H_{S W C T}$ is the height of the SWCT; $h_{\text {avg }}=2.845$ is the average height of the prism blocks. Both measurements are in $\AA$.

The normalized $h e i g h t_{n} \%$ for any prismatic ice phase is defined as follows:

$$
\text { Height }_{n} \%=\frac{N_{n}}{N_{\max }} \times 100
$$

where, $N_{\max }$ is the theoretical maximum possible number of $n$-gonal prism blocks; $N_{n}$ is the actual number of $n$-gonal prism blocks.

Table 2 summarizes the relative proportions of the $n$-gonal prism blocks for the SWCTs. $N_{\max }$ is the same for every $n$, and thus the $(11,0)$ and $(13,0)$ SWCTs have $N_{\max }=63.492 \%$ and $N_{\max }=43.207 \%$, respectively, for all $n$. It is evident that the normalized height percentage matches reasonably well with the occupied volume percentage.

Table 2: Relative proportions of $n$-gonal prism blocks

\begin{tabular}{lllll}
\hline $\begin{array}{l}\text { SWCT } \\
\text { Type }\end{array}$ & $\begin{array}{l}\text { Actual Number } \\
\left(N_{n}\right)\end{array}$ & $\begin{array}{l}\text { Maximum } \\
\text { Number }\left(N_{\max }\right)\end{array}$ & $\begin{array}{l}\text { Normalized Height } \% \\
\left(H_{n}\right)\end{array}$ & Occupied Volume \% $\left(V_{n}\right)$ \\
\hline$(11,0)$ & $N_{4}=40$ & $N_{\max }=63.627$ & $H_{4}=63.492 \%$ & $V_{4}=69.611 \%$
\end{tabular}

SWCT 


\begin{tabular}{lllll}
\hline $\begin{array}{l}\text { SWCT } \\
\text { Type }\end{array}$ & $\begin{array}{l}\text { Actual Number } \\
\left(N_{n}\right)\end{array}$ & $\begin{array}{l}\text { Maximum } \\
\text { Number }\left(N_{\max }\right)\end{array}$ & $\begin{array}{l}\text { Normalized Height } \% \\
\left(H_{n}\right)\end{array}$ & Occupied Volume \% $\left(V_{n}\right)$ \\
\hline$(13,0)$ & $N_{5}=4$ & $N_{\max }=43.207$ & $\begin{array}{l}H_{5}=9.302 \% \\
H_{6}=37.21 \%\end{array}$ & $V_{5}=9.658 \%$ \\
SWCT & $N_{6}=16$ & & $V_{6}=42.982 \%$ \\
\hline
\end{tabular}

\subsubsection{Freezing of an Ice Nanotube}

We track the phase change from the liquid to solid state in water confined within a $(13,0)$ featureless nanotube, approximating a zigzag carbon nanotube. The temperature is lowered in steps of $10 \mathrm{~K}$. Simulations are run in the $N P_{z z} T$ ensemble for $20 \mathrm{~ns}$ at each temperature, subjected to a constant pressure of $0.5 \mathrm{MPa}$ at each temperature for 20 ns.

We observe an abrupt change in phase from the liquid state to ordered prismatic ice phase at a temperature of $280 \mathrm{~K}$. In the previous section, we showed how the height ${ }_{n} \%$ metric matches well with the occupied volume percentage, which has been proven to be an effective order parameter for nucleating quasi-one-dimensional systems [36]. Thus, the phase change of an INT can be described by the height $t_{n} \%$ order parameter. Figure 10 depicts the phase change from the liquid phase, at the beginning of the simulation, to $\approx 60 \%$ hexagonal prismatic phase after the $20 \mathrm{~ns}$ simulation time. The dominant ice phase formed is the hexagonal prismatic phase, with small proportions of pentagonal ice (within 7 height $_{5} \%$ ).

A movie (entitled intEvol.avi in the ESI) documents this phase change.

\section{Conclusion}

d-SEAMS is a flexible post-processing analysis tool, capable of classifying water at both extremes of scale: highly confined systems as well as bulk water. d-SEAMS is the first scientific software to use nix as a build-system to circumvent dependency clashes, along with a YAML-Lua scripting pipeline. The Lua scripting interface, as well as the C++ API, are meant to provide enough rigor for customization, while being easy to use for the general scientific community.

Several applications of qualitative analysis have been presented. We have shown how d-SEAMS is capable of determining the time evolution of structures, from which the growth mechanism and new physical insights have been inferred for heterogenous nucleation. A new order parameter for determining the relative composition of $n$-gonal ice in quasi-one-dimensional nanotubes has been formulated and implemented. The new order parameter, the height\%, produces results that match well with previously defined metrics in the literature. We have demonstrated the versatility of d-SEAMS by performing detailed structural analysis for homogenous nucleation, fMSI formation and ice nanotube freezing from the liquid state.

d-SEAMS (https://dseams.info) is a free and open-source molecular dynamics engine, distributed on GitHub under the GNU General Public License and documented online (https://docs.dseams.info). We envisage future development to incorporate more input formats and structural analysis algorithms. Given the nature of the engine, we expect additions to the framework which would provide insights into biomolecular systems as well.

\section{Acknowledgments}

The computational resources were provided by the HPC facility of the Computer Center(CC), Indian Institute of Technology Kanpur. R.G. acknowledges the invaluable support of his lab PI, D. Goswami.

\section{References}

1. Stukowski, A. Structure Identification Methods for Atomistic Simulations of Crystalline Materials. Modelling and Simulation in Materials Science and Engineering 20, 045021. ISSN: 0965-0393, 1361-651X (June 1, 2012).

2. Carignano, M. A. Formation of Stacking Faults during Ice Growth on Hexagonal and Cubic Substrates. The Journal of Physical Chemistry C 111, 501-504 (Jan. 2007).

3. Herlach, D. M., Palberg, T., Klassen, I., Klein, S. \& Kobold, R. Overview: Experimental studies of crystal nucleation: Metals and colloids. The Journal of Chemical Physics 145, 211703 (Dec. 2016).

4. Woodcock, L. V. Entropy difference between the face-centred cubic and hexagonal close-packed crystal structures. Nature 385, 141-143 (Jan. 1997). 
(a)

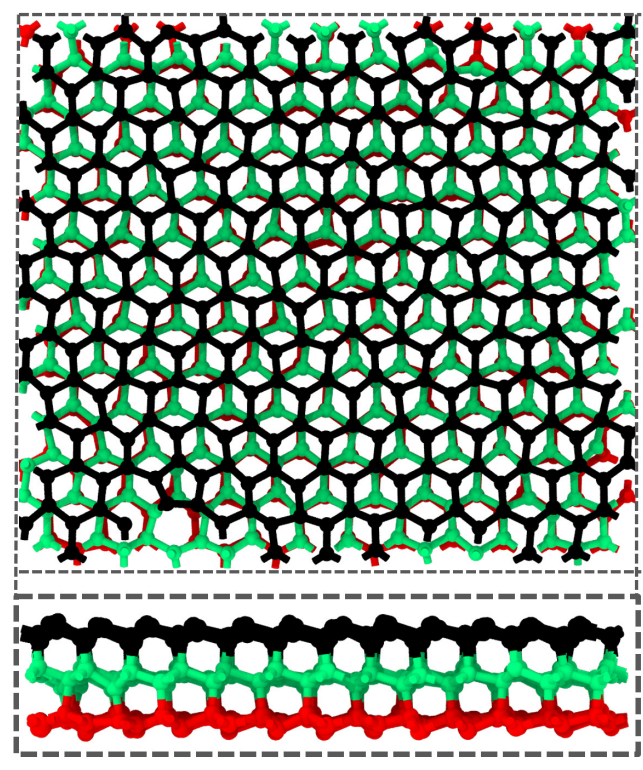

(b)

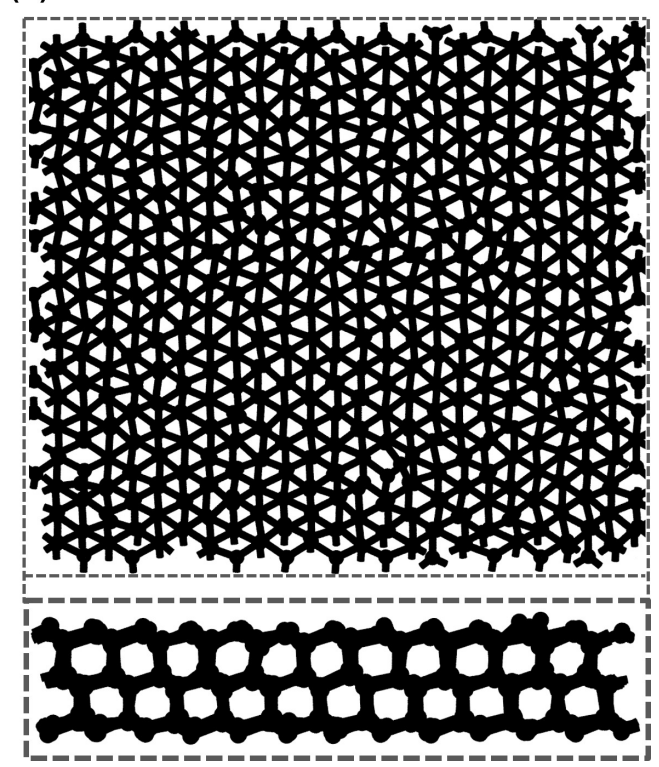

Figure 4: Overhead and side views showing the stacking arrangements of the first two layers of cages on the $\mathrm{AgI}$ surface, when more than half the water has been converted to ice, after more than $\approx 150 \mathrm{~ns}$ of simulation time. (a) The first layer is a layer of HCs. The complete second layer is a layer of DDCs in all cases observed. Mixed rings are sandwiched between the first layer of HCs and the second layer of DDCs. (b) The first and second layers are DDCs. The colour scheme is the same as in the preceding figures.

5. Reinhart, W. F., Long, A. W., Howard, M. P., Ferguson, A. L. \& Panagiotopoulos, A. Z. Machine learning for autonomous crystal structure identification. Soft Matter 13, 4733-4745 (2017).

6. Lupi, L., Hudait, A. \& Molinero, V. Heterogeneous Nucleation of Ice on Carbon Surfaces. Journal of the American Chemical Society 136, 3156-3164 (Feb. 2014).

7. Cox, S. J., Kathmann, S. M., Slater, B. \& Michaelides, A. Molecular simulations of heterogeneous ice nucleation. I. Controlling ice nucleation through surface hydrophilicity. The Journal of Chemical Physics 142, 184704 (May 2015).

8. Bi, Y., Cao, B. \& Li, T. Enhanced heterogeneous ice nucleation by special surface geometry. Nature Communications 8 (May 2017).

9. Petrenko, V. \& Whitworth, R. Physics of Ice. Oxford Univ 2002.

10. Salzmann, C. G., Hallbrucker, A., Finney, J. L. \& Mayer, E. Raman spectroscopic study of hydrogen ordered ice XIII and of its reversible phase transition to disordered ice V. Physical Chemistry Chemical Physics 8, 3088 (2006).

11. Salzmann, C. G., Hallbrucker, A., Finney, J. L. \& Mayer, E. Raman spectroscopic features of hydrogen-ordering in ice XII. Chemical Physics Letters 429, 469-473 (Oct. 2006).

12. Salzmann, C. G., Radaelli, P. G., Mayer, E. \& Finney, J. L. Ice XV: A New Thermodynamically Stable Phase of Ice. Physical Review Letters 103 (Sept. 2009).

13. Falenty, A., Hansen, T. C. \& Kuhs, W. F. Formation and properties of ice XVI obtained by emptying a type sII clathrate hydrate. Nature 516, 231-233 (Dec. 2014).

14. Del Rosso, L., Celli, M. \& Ulivi, L. New porous water ice metastable at atmospheric pressure obtained by emptying a hydrogen-filled ice. Nature Communications 7 (Nov. 2016).

15. Algara-Siller, G. et al. Square ice in graphene nanocapillaries. Nature 519, 443-445 (Mar. 2015).

16. Agrawal, K. V., Shimizu, S., Drahushuk, L. W., Kilcoyne, D. \& Strano, M. S. Observation of extreme phase transition temperatures of water confined inside isolated carbon nanotubes. Nature Nanotechnology 12, 267-273 (Nov. 2016).

17. Zhao, W.-H., Bai, J., Yuan, L.-F., Yang, J. \& Zeng, X. C. Ferroelectric hexagonal and rhombic monolayer ice phases. Chem. Sci. 5, 1757-1764 (2014). 
(a)
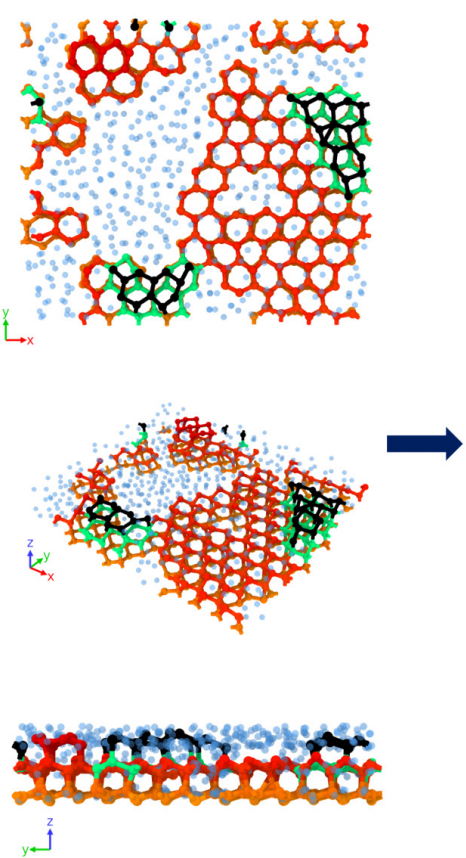

(b)
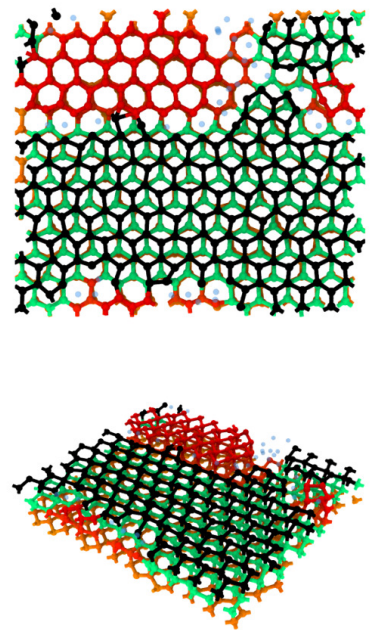

$50009000000 \%$ (c)
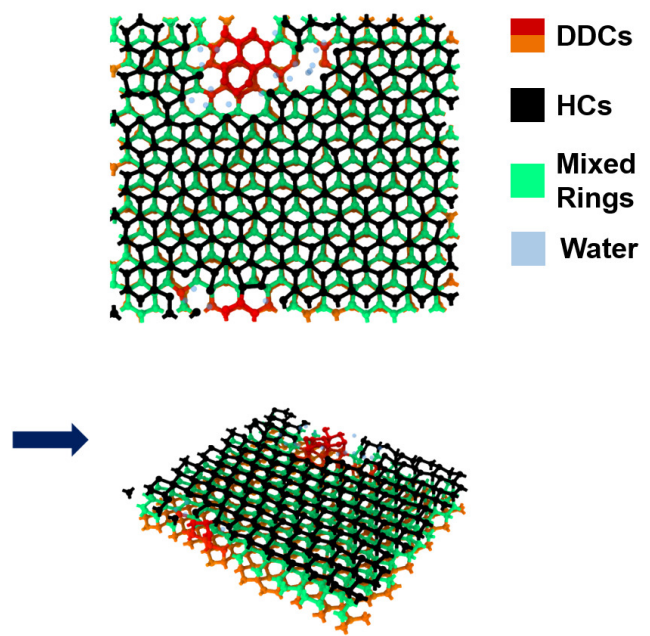

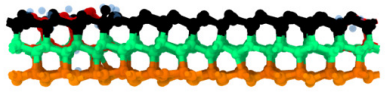

Figure 5: Formation of layers of HCs and DDCs within the first $100 \mathrm{~ns}$ of simulation time. (a) After $\approx 14 n s$, the first layer has not completely formed. (b) After $\approx 60 \mathrm{~ns}$, part of the second layer is comprised of HCs, and a separate portion of the second layer is composed of DDCs. The part of the second layer made up of HCs is distinguishable in the top view because of the AA-stacking order of HCs on the first layer of HCs. (c) After $\approx 100 \mathrm{~ns}$, the second layer is almost entirely composed of DDCs. The colour scheme is shown in the legend. DDCs are coloured in shades of yellow to vermilion, according to the distance from the AgI surface.
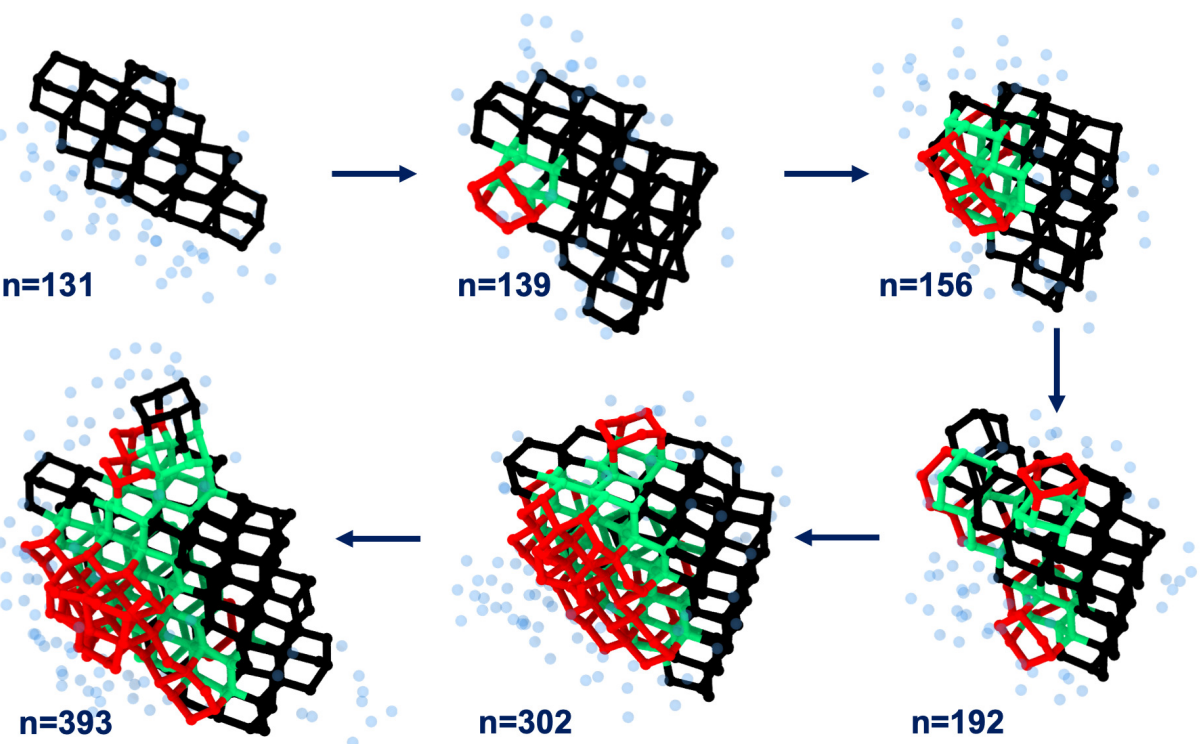

Figure 6: Evolution of an ice crystallite of $\mathrm{mW}$ water at $208 \mathrm{GPa}$ in the NPT ensemble. DDCs are in black, HCs are in red and mixed rings belonging to both DDCs and HCs are in green. The number of particles in the largest cluster $n$ is shown for every snapshot of the trajectory. Water molecules which do not participate in the cages are in transparent blue. 

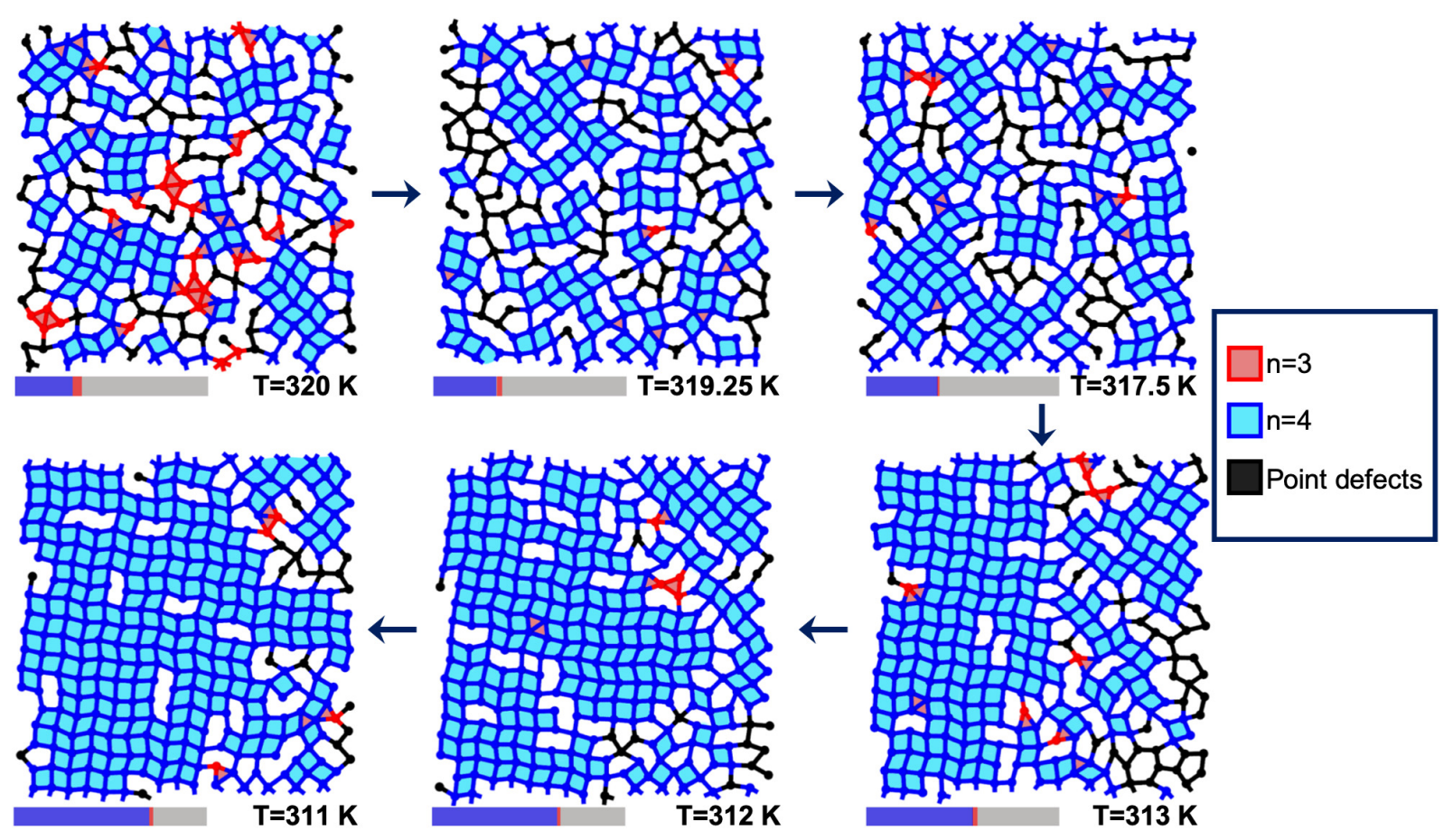

Figure 7: Snapshots of monolayer water being cooled from $320 \mathrm{~K}$ to $310 \mathrm{~K}$, at $1 \mathrm{GPa}$. The stacked bars in the lower left corner of each snapshot denote the coverage area percentages for the 4-membered rings (blue) and 3-membered rings (red). The colours of the particles, bonds and interior ring shading are shown in the legend.

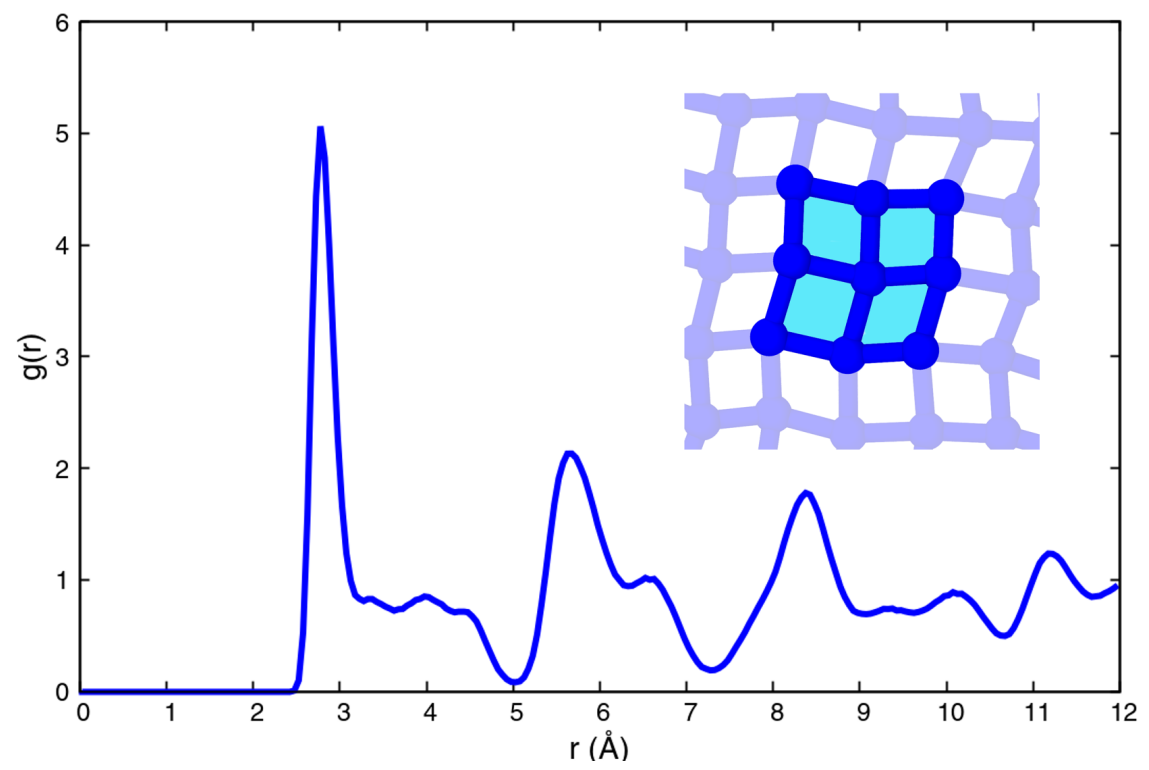

Figure 8: In-plane oxygen-oxygen radial distribution function of the ice formed at $310 \mathrm{~K}$ and $P_{x x}=1 G P a$. The inset shows the 4-membered rings characteristic of fMSI. 

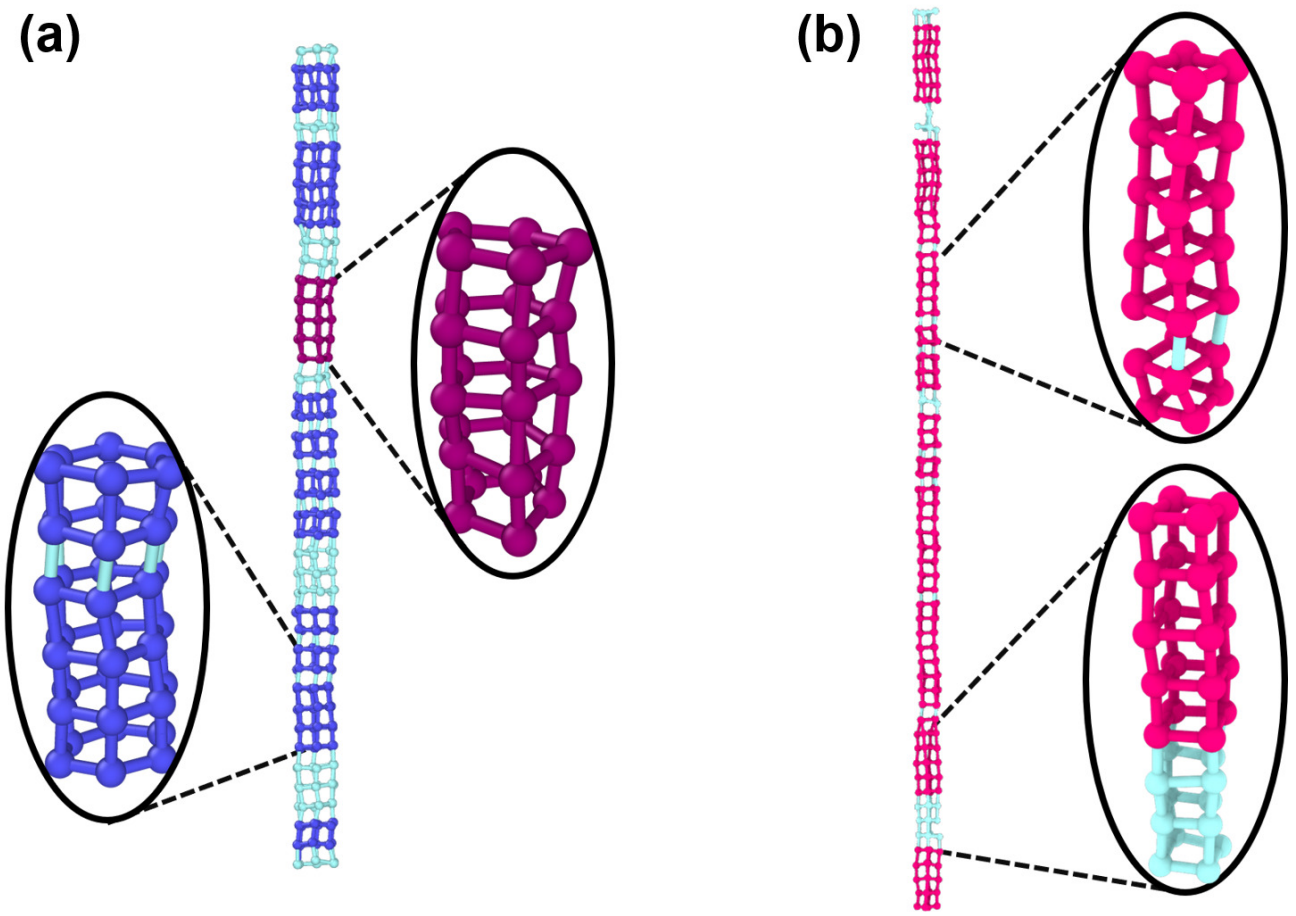

Figure 9: View of ice structures at 240K confined within (a) $(13,0)$ zig-zag nanotube at $0.1 \mathrm{MPa}$ and (b) (11,0) zig-zag nanotube at $1 \mathrm{MPa}$. The unclassified phase, either water or deformed prism blocks, is sea-green. Prism blocks of pentagonal, hexagonal, and tetragonal ices are shown in purple, blue and pink respectively.

18. Takaiwa, D., Hatano, I., Koga, K. \& Tanaka, H. Phase diagram of water in carbon nanotubes. Proceedings of the National Academy of Sciences 105, 39-43 (Dec. 2007).

19. Bai, J., Angell, C. A. \& Zeng, X. C. Guest-free monolayer clathrate and its coexistence with two-dimensional high-density ice. Proceedings of the National Academy of Sciences 107, 5718-5722 (Mar. 2010).

20. Salzmann, C. G., Radaelli, P. G., Slater, B. \& Finney, J. L. The polymorphism of ice: five unresolved questions. Physical Chemistry Chemical Physics 13, 18468 (2011).

21. Salzmann, C. G. Advances in the experimental exploration of water's phase diagram. The Journal of Chemical Physics 150, 060901 (Feb. 2019).

22. Chen, J., Schusteritsch, G., Pickard, C. J., Salzmann, C. G. \& Michaelides, A. Two Dimensional Ice from First Principles: Structures and Phase Transitions. Physical Review Letters 116 (Jan. 2016).

23. Koga, K., Tanaka, H. \& Zeng, X. C. First-order transition in confined water between high-density liquid and low-density amorphous phases. Nature 408, 564-567 (Nov. 2000).

24. Zangi, R. \& Mark, A. E. Monolayer Ice. Physical Review Letters 91 (July 2003).

25. Bai, J. \& Zeng, X. C. Polymorphism and polyamorphism in bilayer water confined to slit nanopore under high pressure. Proceedings of the National Academy of Sciences 109, 21240-21245 (Dec. 2012).

26. Zhu, Y., Wang, F., Bai, J., Zeng, X. C. \& Wu, H. Compression Limit of Two-Dimensional Water Constrained in Graphene Nanocapillaries. ACS Nano 9, 12197-12204. ISSN: 1936-0851 (Dec. 22, 2015).

27. Zhu, Y., Wang, F., Bai, J., Zeng, X. C. \& Wu, H. Formation of Trilayer Ices in Graphene Nanocapillaries under High Lateral Pressure. The Journal of Physical Chemistry C 120, 8109-8115 (Apr. 2016).

28. Gao, Z., Giovambattista, N. \& Sahin, O. Phase Diagram of Water Confined by Graphene. Sci Rep 8, 1-11. ISSN: 2045-2322 (Apr. 18, 2018).

29. Boettiger, C. An introduction to Docker for reproducible research. ACM SIGOPS Operating Systems Review 49, 71-79 (Jan. 2015).

30. Dolstra, E., de Jonge, M. \& Visser, E. Nix: A Safe and Policy-Free System for Software Deployment, 15 (2004).

31. Prins, J., Suresh, J. \& Dolstra, E. Nix fixes depency hell on all Linux distributions in Linux (2008). 


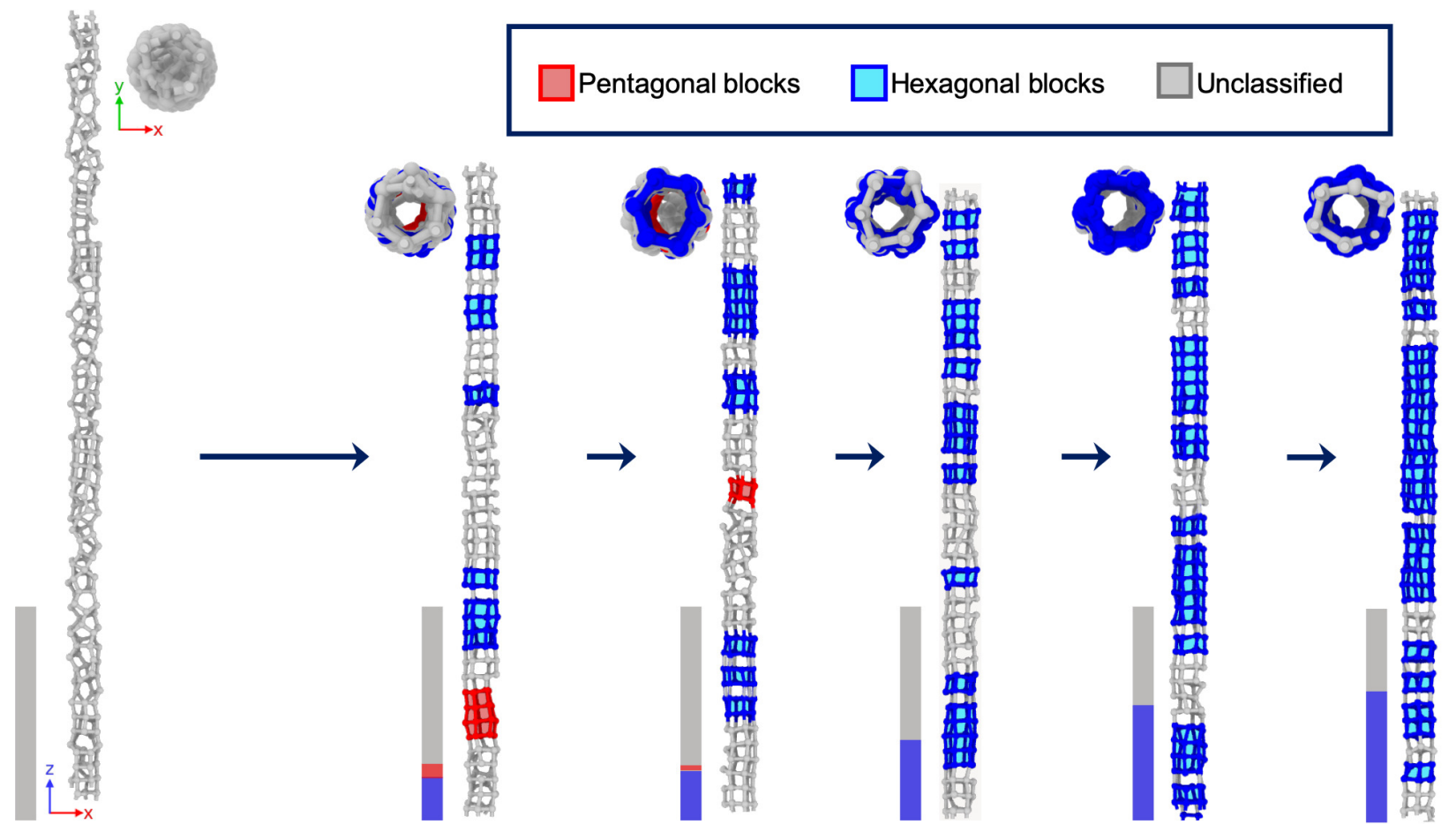

Figure 10: Snapshots of an icy nanoribbon at various timesteps, at $280 K$ and $P_{z z}=0.5 M P a$, in a (13, 0$)$ smooth single-walled nanotube. The stacked bars in the lower left corner of each snapshot denote the height percentages for the dominant phases: hexagonal prism blocks (blue) and pentagonal prism blocks (red). Initially, the quasi-one-dimensional water is entirely in the liquid phase.

32. Ten Wolde, P. R., Ruiz-Montero, M. J. \& Frenkel, D. Numerical calculation of the rate of crystal nucleation in a Lennard-Jones system at moderate undercooling. The Journal of Chemical Physics 104, 9932-9947 (June 1996).

33. Li, T., Donadio, D., Russo, G. \& Galli, G. Homogeneous Ice Nucleation from Supercooled Water. Physical Chemistry Chemical Physics 13, 19807. ISSN: 1463-9076, 1463-9084 (2011).

34. Franzblau, D. S. Computation of ring statistics for network models of solids. Physical Review B 44, 4925-4930 (Sept. 1991).

35. Haji-Akbari, A. \& Debenedetti, P. G. Direct Calculation of Ice Homogeneous Nucleation Rate for a Molecular Model of Water. Proceedings of the National Academy of Sciences 112, 10582-10588. ISSN: 0027-8424, 10916490 (Aug. 25, 2015).

36. Goswami, A. \& Singh, J. K. A General Topological Network Criterion for Exploring the Structure of Icy Nanoribbons and Monolayers. Physical Chemistry Chemical Physics (2019).

37. Steinhardt, P. J., Nelson, D. R. \& Ronchetti, M. Bond-orientational order in liquids and glasses. Physical Review $B$ 28, 784-805 (July 1983).

38. Moore, E. B., de la Llave, E., Welke, K., Scherlis, D. A. \& Molinero, V. Freezing, Melting and Structure of Ice in a Hydrophilic Nanopore. Physical Chemistry Chemical Physics 12, 4124. ISSN: 1463-9076, 1463-9084 (2010).

39. Nguyen, A. H. \& Molinero, V. Identification of Clathrate Hydrates, Hexagonal Ice, Cubic Ice, and Liquid Water in Simulations: The CHILL+ Algorithm. The Journal of Physical Chemistry B 119, 9369-9376. ISSN: 1520-6106, 1520-5207 (July 23, 2015).

40. Stukowski, A. Visualization and analysis of atomistic simulation data with OVITO-the Open Visualization Tool. Modelling and Simulation in Materials Science and Engineering 18, 015012 (Dec. 2009).

41. Humphrey, W., Dalke, A. \& Schulten, K. VMD: Visual molecular dynamics. Journal of Molecular Graphics 14, 33-38 (Feb. 1996).

42. Dolstra, E., Löh, A. \& Pierron, N. NixOS: A Purely Functional Linux Distribution. Journal of Functional Programming 20, 577-615. ISSN: 1469-7653, 0956-7968 (Nov. 2010). 
43. Peng, R. D. Reproducible Research in Computational Science. Science 334, 1226-1227. ISSN: 0036-8075, 1095-9203 (Dec. 2, 2011).

44. Shirts, M. R. et al. Lessons learned from comparing molecular dynamics engines on the SAMPL5 dataset. Journal of Computer-Aided Molecular Design 31, 147-161 (Oct. 2016).

45. Stoddard, S. D. Identifying clusters in computer experiments on systems of particles. Journal of Computational Physics 27, 291-293 (May 1978).

46. Vonnegut, B. The Nucleation of Ice Formation by Silver Iodide. Journal of Applied Physics 18, 593-595 (July 1947).

47. Zielke, S. A., Bertram, A. K. \& Patey, G. N. A Molecular Mechanism of Ice Nucleation on Model AgI Surfaces. The Journal of Physical Chemistry B 119, 9049-9055 (Oct. 2014).

48. Abascal, J. L. F., Sanz, E., Fernández, R. G. \& Vega, C. A potential model for the study of ices and amorphous water: TIP4P/Ice. The Journal of Chemical Physics 122, 234511 (June 2005).

49. Prerna, Goswami, R., Metya, A. K., Shevkunov, S. V. \& Singh, J. K. Study of Ice Nucleation on Silver Iodide Surface with Defects. Molecular Physics, 1-13. ISSN: 0026-8976, 1362-3028 (Aug. 25, 2019).

50. Zielke, S. A., Bertram, A. K. \& Patey, G. N. Simulations of Ice Nucleation by Model AgI Disks and Plates. The Journal of Physical Chemistry B 120, 2291-2299 (Feb. 2016).

51. Molinero, V. \& Moore, E. B. Water Modeled As an Intermediate Element between Carbon and Silicon†. The Journal of Physical Chemistry B 113, 4008-4016 (Apr. 2009).

52. Yang, L., Guo, Y. \& Diao, D. Structure and dynamics of water confined in a graphene nanochannel under gigapascal high pressure: dependence of friction on pressure and confinement. Physical Chemistry Chemical Physics 19, 14048-14054 (2017). 


\title{
Supporting information for:
}

\section{d-SEAMS: Deferred Structural Elucidation Analysis for Molecular Simulations}

\author{
Rohit Goswami, ${ }^{\dagger, \dagger}$ Amrita Goswami, ${ }^{\ddagger}, \mathbb{T}$ and Jayant K. Singh ${ }^{*, \ddagger}$ \\ $\dagger$ Currently at the Department of Chemistry, Indian Institute of Technology Kanpur \\ $\ddagger$ Department of Chemical Engineering, Indian Institute of Technology Kanpur \\ \Contributed equally to this work \\ E-mail: jayantks@iitk.ac.in
}

\section{Design}

The d-SEAMS framework is designed to be accessible to the end-user, while offering a powerful system of building blocks and generics for extensions. The engine itself is written in $\mathrm{C}++$ and is compiled to a binary. This binary accepts Lua input scripts to expose the functionality of the software such that the underlying data-structures and computations are abstracted away from the user. To facilitate reproduction of results and to prevent users from accessing conflicting or unphysical functional manipulations of the input data, YAML options mask certain functions from being exposed. The YAML workflows are completely reproducible in Lua scripts, but provide an easy way to share methodologies and also reduces the cognitive load of going through the complete API documentation. 

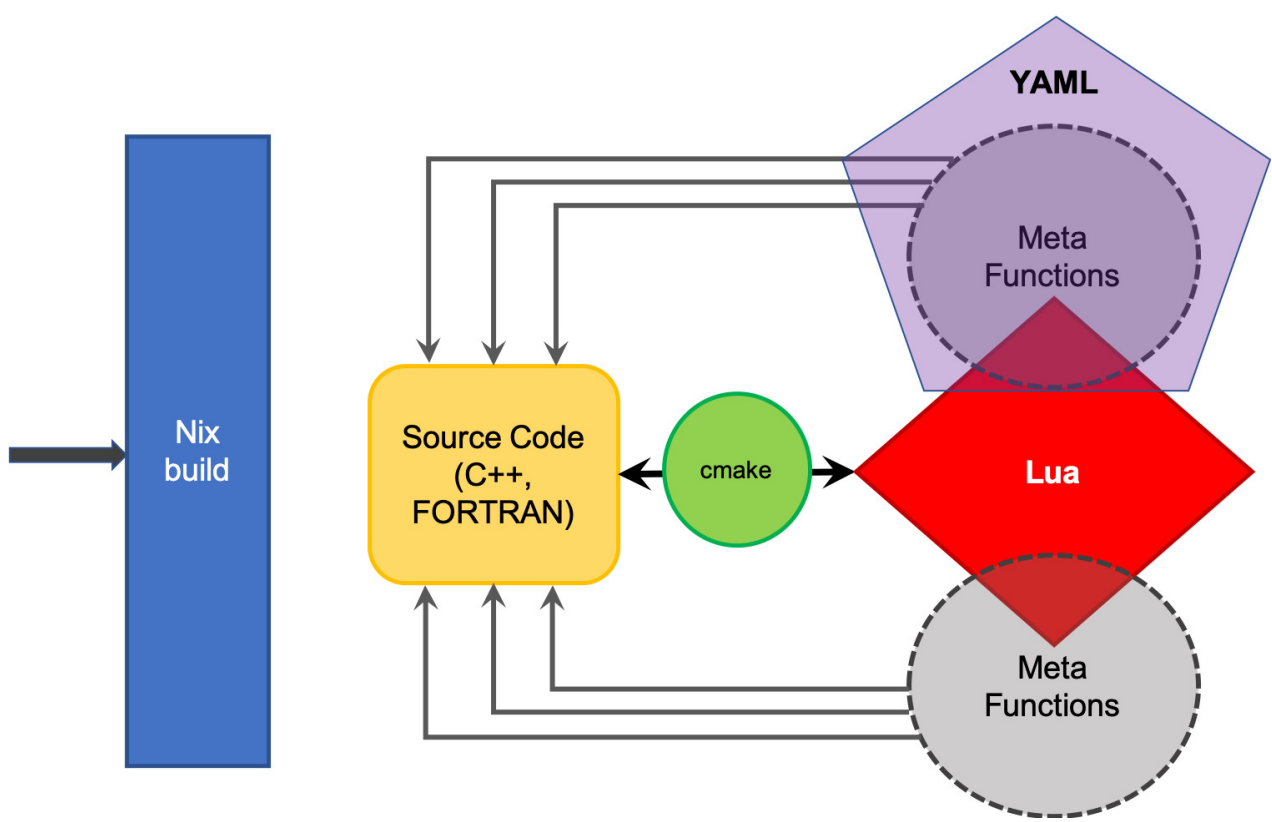

Figure S1: Work-flow of d-SEAMS. 'Nix' uses cryptographic hashes to ensure reproducible builds over all systems. 'Cmake' compiles and builds the source code, using the dependencies managed by "nix'. The 'Lua' script provides an interface to the back-end functions. Combinations of these ' $\mathrm{C}++{ }^{\text {' }}$ functions can be called the 'meta' functions. The 'YAML' interface exposes only relevant back-end and 'Lua' functions, corresponding to the user-determined pre-determined work-flow.

Figure S1 is a schematic of the overall design architecture of d-SEAMS. The Lua scripting interface exposes C-like functions to create custom work-flows. The YAML configuration file provides options for pre-determined work-flows. Users with different requirements and experience can interact with d-SEAMS. The three main components of the code architecture are enumerated below:

- YAML configuration file: This contains options for pre-determined work-flows. For example, a user who enters the option for the confined quasi-one-dimensional ice determination will only be exposed to the relevant functions for INT prism determination. Multiple workflows can be selected at the same time.

- Lua Interface: $\mathrm{C}++$ functions are registered as Lua functions, which are called from a Lua script. Lua is a C-like scripting language, enabling users to call the Lua functions without needing to learn a software-specific scripting convention. The advantage of 
using Lua over directly calling $\mathrm{C}++$ functions is that the users need not be concerned with pointers and clean-up of the $\mathrm{C}++$ structures. The Lua language also has a rich set of cross platform extensions for file handling, and is also supported by major editors for syntax highlighting.

- $\mathrm{C}++$ Back-end: The back-end is written in modern $\mathrm{C}++$, employing common data structures, used uniformly throughout the code. Users can easily extend and write their own $\mathrm{C}++$ header files, and the documentation covers manipulating the build system to accept both user-defined and external headers. Registering custom $\mathrm{C}++$ functions as Lua functions, to be subsequently called in Lua scripts, is also documented. GDB ${ }^{\mathrm{S} 1}$ can be used for code debugging, since the back-end is in $\mathrm{C}++$.

From a user perspective, we have designed the Lua functions to mimic the mindset of a computational chemist, without burdening them with the software implementation. We have also ensured reproducibility, both as an aid to the science ${ }^{\mathrm{S} 2}$ intended and also to allow for bugs to be dealt with more efficiently. This reproducibility is ensured during build, compile, and linking stages, by leveraging the functional, immutable binaries produced by nix. ${ }^{\mathrm{S} 3}$ The dependencies are handled reproducibly, though for ease of extension by the wider community, most of the build system is in CMake. We use nix to ensure that the dependencies of the binary are fully reproducible, as a consequence of traversing the build graph defined by the nix-derivation. The binary itself has a server-client architecture, to ensure that the user can transparently interact with the code without needing a background in functional programming. Since the backend server is written entirely in modern $\mathrm{C}++$, the GDB debugger is usable throughout. The server-client nature of the system, though currently a bottleneck in terms of parallelism, allows for a single compiled binary to be used for the execution of multiple different Lua input scripts, with each script spawning a separate process. 


\section{Nix}

The nix derivation provides a deterministic package-level lock on all dependencies and is written in nix, a lazy, dynamically typed, purely functional language. ${ }^{\mathrm{S} 4}$ This choice of distribution also allows the user to extend the system reproducibly, ensuring that changes can be quickly merged in-to the upstream repository. More details of the "nix'-build system are described in the main text of the manuscript.

\section{Lua}

Existing molecular dynamics packages suffer from not having design parameters built-in, and with time, this has led to unique and non-standard syntax being used, as seen in the input scripts of LAMMPS ${ }^{\mathrm{S} 5}$ and GROMACS, ${ }^{\mathrm{S} 6}$ amongst others. Popular text editors do not offer syntax highlighting for these custom non-standard and software-specific syntaxes. For such software-specific syntaxes, the code is unusable without learning from the documentation. An alternative to crafting a new input script syntax for each software, is to use python for scripting. ${ }^{\text {S7 }}$ However, the version dependence of each internal segment can become intractable without continuous development, and as a result, these spawn multiple language-specific errors, and they work best only on the Linux distribution on which the creators have worked . Furthermore, we assert that the proliferation of python scripts and the odd-ease at which they may be, in theory mixed and matched, in practice causes many clashes, for example, EsPreSSo $^{\mathrm{S} 8}$ and Quantum EsPreSSo ${ }^{\mathrm{S} 9}$ (and more generally, Scipy ${ }^{\mathrm{S} 10}$ and Numpy ${ }^{\mathrm{S} 11}$ ) have several function names in common which cause difficult to debug when used together in an input script. Also, python has less support for debugging, and the language server support is lacking, making complex python code difficult to debug. This is partially due to the design of python itself. The lack of static typing has the effect of making the compiler oblivious to bugs which are caught easily in statically typed languages like FORTRAN and C++.

To address these concerns we have opted to use Lua as the scripting interface, which has C-like functions. It is widely supported in terms of syntax highlighting, and is easy to 
interface with $\mathrm{C}++$ code. Furthermore, the error handling is such that it is amenable for arbitrarily complex GDB debugger workflows, ${ }^{\mathrm{S} 12}$ and the rich standard library of Lua, along with user extensions, have no clashes. Lua is also user friendly due to its C-like syntax. The rich table and object handling makes writing out image data easy. Furthermore, though Lua was released two years after python was first introduced (1993 and 1991, respectively) unlike python, which is still transitioning from the major API change of two to three, the Lua API is stable, mostly because it has been designed to be an embedded language and not a general-purpose language like python. Lua has been the darling of the gaming development community, and has proven its worth in many related domains such as image handling. Apart from the user-friendly helper functions, our design has the Lua interface, which offers every core function to the user. This permits arbitarily complicated workflows to be used without re-compiliation, which is a boon for HPC cluster usage. We recommend strongly in the docs, that foreign code, once interfaced to the $\mathrm{C}++$ engine, should be bound in Lua for the end-users as well.

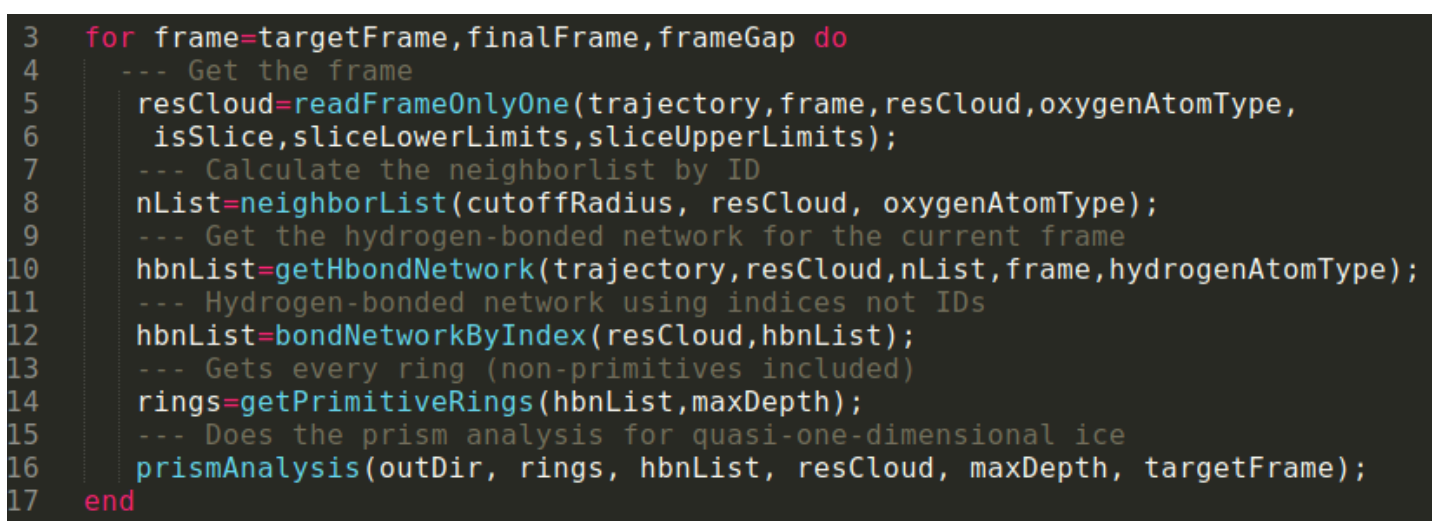

Figure S2: The 'lua' input script, where the user is able to call any of the functions not voided by the options in the 'YAML' file.

Figure S2 shows a typical Lua input script, which calls functions exposed by the current YAML file work-flow. 


\section{YAML}

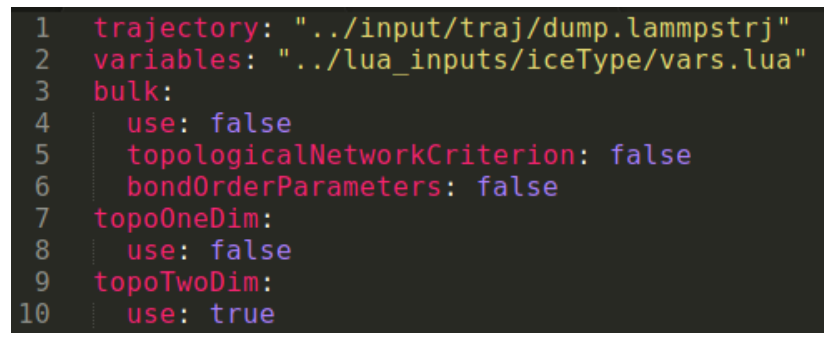

Figure S3: The 'YAML' file, where boolean values are set to restrict functions exposed to the 'lua' scripting engine.

To improve usability and reduce the time required reading the API documentation, we have split the usage into a unique YAML-Lua design. The Lua interface is for power users, however, to reduce mistakes, options set in the YAML files will deactivate certain functions, in order to prevent incorrect manipulations of the internal data-structures. The YAML interface diverts the flow of functionality and code to different paths, and thus different algorithms. This also prevents name-clashes of similar functions for mutually exclusive work-flows. For example, an input system can either be a bulk system, a quasi-one-dimensional system or a quasi-two-dimensional system. The YAML file offers truthy options, an example of which is shown in Figure S3, and subsequently masks functions not applicable for the given system type.

\section{References}

(S1) Stallman, R.; Pesch, R.; Shebs, S., et al. Debugging with GDB. Free Software Foundation 2002, 51, 02110-1301.

(S2) Mesirov, J. P. Accessible Reproducible Research. 327, 415-416.

(S3) Dolstra, E.; family=Jonge, p. u., given=Merijn; Visser, E. Nix: A Safe and Policy-Free System for Software Deployment. 15. 
(S4) Dolstra, E.; Lh, A.; Pierron, N. NixOS: A Purely Functional Linux Distribution. 20, $577-615$.

(S5) Plimpton, S. Fast Parallel Algorithms for ShortRange Molecular Dynamics. 42.

(S6) Abraham, M. J.; Murtola, T.; Schulz, R.; Pll, S.; Smith, J. C.; Hess, B.; Lindahl, E. GROMACS: High Performance Molecular Simulations through Multi-Level Parallelism from Laptops to Supercomputers. 1-2, 19-25.

(S7) McGibbon, R. T.; Beauchamp, K. A.; Harrigan, M. P.; Klein, C.; Swails, J. M.; Hernndez, C. X.; Schwantes, C. R.; Wang, L.-P.; Lane, T. J.; Pande, V. S. MDTraj: A Modern Open Library for the Analysis of Molecular Dynamics Trajectories. 109, $1528-1532$.

(S8) Weik, F.; Weeber, R.; Szuttor, K.; Breitsprecher, K.; family=Graaf, p. u., given=Joost; Kuron, M.; Landsgesell, J.; Menke, H.; Sean, D.; Holm, C. ESPResSo 4.0 an Extensible Software Package for Simulating Soft Matter Systems. 227, 1789-1816.

(S9) Giannozzi, P.; Baroni, S.; Bonini, N.; Calandra, M.; Car, R.; Cavazzoni, C.; Ceresoli, D.; Chiarotti, G. L.; Cococcioni, M.; Dabo, I.; Corso, A. D.; family=Gironcoli, p. u., given=Stefano; Fabris, S.; Fratesi, G.; Gebauer, R.; Gerstmann, U.; Gougoussis, C.; Kokalj, A.; Lazzeri, M.; Martin-Samos, L.; Marzari, N.; Mauri, F.; Mazzarello, R.; Paolini, S.; Pasquarello, A.; Paulatto, L.; Sbraccia, C.; Scandolo, S.; Sclauzero, G.; Seitsonen, A. P.; Smogunov, A.; Umari, P.; Wentzcovitch, R. M. QUANTUM ESPRESSO: A Modular and Open-Source Software Project for Quantum Simulations of Materials. 21, 395502.

(S10) Oliphant, T. E. Python for Scientific Computing. 9, 10-20.

(S11) family=Walt, p. d. u., given=S.; Colbert, S. C.; Varoquaux, G. The NumPy Array: A Structure for Efficient Numerical Computation. 13, 22-30. 
(S12) Stallman, R.; Pesch, R.; Shebs, S. Debugging with GDB. 352. 